\title{
Inaugural Breakthrough Prize in Mathematics Awarded
}

Five mathematicians have been selected as recipients of the first Breakthrough Prize in Mathematics by the Breakthrough Prize Foundation.

SIMON DONALDSON of Stony Brook University

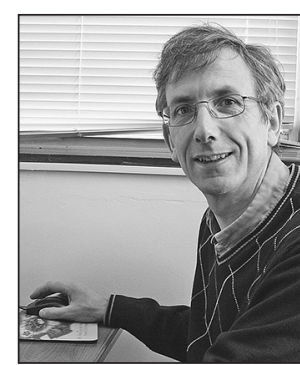

Simon Donaldson

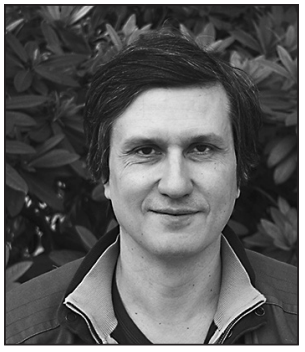

Maxim Kontsevich

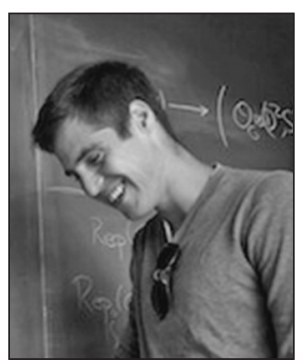

Jacob Lurie and Imperial College London was honored "for the new revolutionary invariants of 4-dimensional manifolds and for the study of the relation between stability in algebraic geometry and in global differential geometry, both for bundles and for Fano varieties."

MAXIM KonTSEvich of the Institut des Hautes Études Scientifiques was recognized "for work making a deep impact in a vast variety of mathematical disciplines, including algebraic geometry, deformation theory, symplectic topology, homologisystems."

JACOB LURIE of Harvard University was selected "for his work on the foundations of higher category theory and derived algebraic geometry; for the classification of fully extended topological quantum field theories; and for providing a moduli-theoretic interpretation of elliptic cohomology." cal algebra, and dynamical

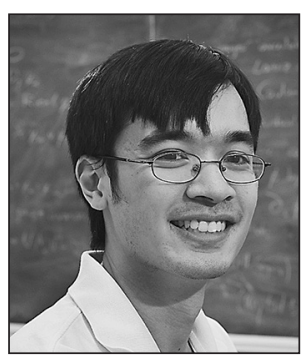

TERENCE TAO of the University of California Los Angeles, was honored "for numerous breakthrough contributions to harmonic analysis, combinatorics, partial differential equations, and analytic number theory."

RICHARD TAYLOR of the Terence Tao

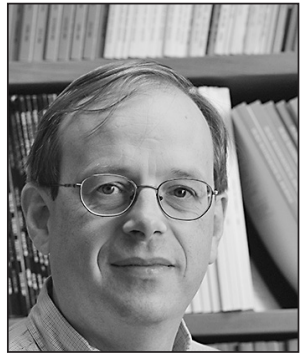
Institute for Advanced Study was recognized "for numerous breakthrough results in the theory of automorphic forms, including the Taniyama-Weil conjecture, the local Langlands conjecture for general linear groups, and the Sato-Tate conjecture."

The Breakthrough Prize in Mathematics was created by Mark Zuckerberg and Yuri Milner in 2013. It aims to recRichard Taylor ognize major advances in the field, to honor the world's best mathematicians, to support their future endeavors, and to communicate the excitement of mathematics to the general public. The Breakthrough Prize carries a cash award of US\$3 million.

The prizewinners will serve on the selection committee responsible for choosing subsequent winners of the prize from the pool of contenders nominated by the mathematics community. From 2015 on, one Breakthrough Prize in Mathematics will be awarded every year.

Photo credits: Simon Donaldson-Imperial College, London; Maxim Kontsevich-MCV/IHES; Jacob Lurie-Harvard University Department of Mathematics; Terence Tao-Photo by Kyle Alexander; Richard Taylor-Photo by Cliff Moore. 